\title{
Stochastic to Deterministic Crossover of Fractal Dimensions for a Langevin Equation
}

\author{
David A. Egolf \\ and \\ Henry S. Greenside $\circledast$ \\ Department of Physics and \\ Center for Nonlinear and Complex Systems \\ Duke University, Durham, NC 27706 \\ dae@phy.duke.edu; hsg@cs.duke.edu
}

\begin{abstract}
Using algorithms of Higuchi and of Grassberger and Procaccia, we study numerically how fractal dimensions cross over from finite-dimensional Brownian noise at short time scales to finite values of deterministic chaos at longer time scales for data generated from a Langevin equation that has a strange attractor in the limit of zero noise. Our results suggest that the crossover occurs at such short time scales that there is little chance of finite-dimensional Brownian noise being incorrectly identified as deterministic chaos.
\end{abstract}

2.50.+s, 5.40.+j, 5.45.+b, 47.25.Mr

Typeset Using REVTEX 
A significant recent effort in nonlinear dynamics has been devoted to analyzing time series so as to determine whether they are chaotic or stochastic [1]. Chaotic time series arise from deterministic strange attractors whose underlying geometric structure in state space can be used to improve short term forecasting [1]], to remove noise [1], or to stabilize unstable periodic orbits by small perturbations of parameters [2]. Stochastic time series such as Gaussian white noise or Brownian motion can not be obtained from any finite set of deterministic equations, and are less amenable to prediction and control.

An early expectation was that fractal dimensions of time series would provide a tool for distinguishing chaotic from stochastic behavior. A small empirical fractal dimension would correspond to a deterministic chaotic time series while a large or diverging fractal dimension (as a function of embedding dimension) would correspond to a stochastic time series [3:41. This conjecture was supported by some numerical experiments, e.g., the work of Ben-Mizrachi et al [5] which showed a crossover between high-dimensional white noise and low-dimensional chaos for time series generated by a chaotic map with additive white noise. The crossover occurred as a function of distance between points in phase space, with large correlation dimensions $\nu$ (increasing linearly with the embedding dimension) occurring at small distances and a finite fractal dimension (independent of embedding dimension) occurring at larger distances.

Several recent papers [6, [7,8] have pointed out that this conjecture - that a fractal dimension can distinguish deterministic from stochastic mechanisms - is not strictly correct. Osborne and Provenzale [G] showed empirically that stochastic time series with a power-law power spectrum, $P(\omega) \propto \omega^{\alpha}$, yield a finite fractal dimension $D$ that is parameterized by the index $\alpha$; the precise value of $D$ depends on some details of the random phases used in generating the time series [8]. Theiler has pointed out that such time series are not likely to be identified incorrectly as deterministic if observed in experimental data [7]. This follows since time series with a power-law power spectrum have long correlation times that scale with the length of the time series, and so are easily identified in the Grassberger-Procaccia dimension algorithm [9] by rejecting points that are close in phase space and also close in 
time [10].

In this Brief Report, we explore further the extent to which stochastic Brownian motions can be distinguished from chaotic behavior using dimension calculations. Our starting point is the observation that the calculation of Mizrachi et al does not include the important case of a noisy dynamical system that evolves continuously in time such as a Langevin equation. Unlike a noisy map, the paths generated by noisy vector Langevin equations have a finite fractal dimension in the limit of short-time scales, with Hausdorff dimension $D=2$ [6, 11]. Over longer but still finite time scales (long compared to the correlation time of the time series), low-amplitude noise simply blurs the structure of the strange attractor and a finite fractal dimension is found, close to the dimension of the unperturbed attractor.

There is then the possibility of an interesting crossover from finite-dimensional stochastic behavior to finite-dimensional deterministic behavior for continuous-time noisy systems. In the following, we study this crossover numerically and find that it occurs at such short time scales (for fixed moderate amounts of noise) that it is unlikely that the two effects will be confused in empirical data. The results below also confirm some scaling predictions made by Theiler in his analysis of fractional Brownian motions [7].

In our numerical experiments, we focus our attention on a vector Langevin equation of the form:

$$
\mathbf{X}^{\prime}(t)=\mathbf{F}(\mathbf{X})+\epsilon \mathbf{G}(t)
$$

where the vector field $\mathbf{F}(\mathbf{X})$ is smooth and where the vector $\mathbf{G}$ represents a delta-function correlated Gaussian white noise source, $\left\langle G_{i}(t) G_{j}\left(t^{\prime}\right)\right\rangle=\delta_{i, j} \delta\left(t-t^{\prime}\right)$. In the limit of large noise strength $\epsilon \gg 1$, we can ignore the field $\mathbf{F}$ and we obtain a Brownian-noise process whose fractal dimension is $D=2$ [11]. In the opposing limit of small noise strength $\epsilon \ll 1$, the Langevin equation reduces to a deterministic set of ordinary differential equations. To study a possible crossover in fractal dimension as the noise strength $\epsilon$ is varied, we choose the vector field $\mathbf{F}$ to be a four-dimensional chaotic model derived by Lorenz [12]

$$
F_{1}=X_{2}\left(X_{3}-X_{4}\right)-X_{1}+c
$$




$$
\begin{aligned}
& F_{2}=X_{3}\left(X_{4}-X_{1}\right)-X_{2}+c, \\
& F_{3}=X_{4}\left(X_{1}-X_{2}\right)-X_{3}+c, \\
& F_{4}=X_{1}\left(X_{2}-X_{3}\right)-X_{4}+c .
\end{aligned}
$$

For the parameter value $c=100$, these equations have chaotic solutions with a fractal dimension $D \approx 3.3[12$. This dimension is distinctly larger than the value for Brownian paths.

We expect the Langevin equation Eq. 1 to have at least four different dimension scaling regimes in the general case, with the size of each regimes depending on the noise strength $\epsilon$, the length of the time integration $T$, and details of the strange attractor. For zero noise strength $\epsilon=0$, we expect a scaling regime with dimension $D=1$ at short time scales and a regime of $D=3.3$ at long time scales compared to the correlation time. The former corresponds to the locally linear geometry of any smooth path in phase space. When noise is present, a third scaling regime with $D=2$ (for Gaussian noise) should be visible at sufficiently short time scales. This new regimes arises from the scale-invariant non-differentiable structure of a Brownian path. For fixed noise and for sufficiently long time series, a fourth scaling regime with $D \rightarrow \infty$ should appear, representing the fact that the path eventually fills all of space.

We have integrated numerically the Langevin equation Eq. 1 with the vector field Eq. 2 for different noise strengths $\epsilon$ and lengths of time series $N$. We used a simple secondorder accurate stochastic Runge-Kutta algorithm [13], and have verified that our results are not sensitive to the choice of time step $\triangle t$. Estimates of the correlation dimension $\nu$ were obtained by delay-embeddings of time series of the first variable $X_{1}(t)$ into spaces of successively higher dimensions $e$, with an embedding delay of order the empirical correlation $\tau_{c} \approx 0.1$. Although the state vector $\mathbf{X}$ is known directly from Eq. [ so that embedding is not strictly necessary, scaling regimes are identified more easily since they are invariant under increasing embedding dimension $e$. In our calculations, we also make the simplifying assumption that there is no observational noise, a complication that should be examined at 
a future date.

Our results are presented in Figures 1-3. For zero additive noise $\epsilon=0$, Fig. 11 shows the local slope $\nu$ (correlation dimension) of scaling curves obtained with an approximate Grassberger-Procaccia algorithm, in which a sample of 1000 points is chosen from the time series and then all distances are calculated between each of these points and every other point in the entire time series. The 1000 points were chosen to be approximately evenly spaced throughout the time series. This method yielded similar results to Theiler's boxassisted method [14], in which all distances between points in phase space are calculated for points that are sufficiently close. We do not implement Theiler's idea [10] of throwing out state-space points that are close in time, since these points give valuable information about possible stochastic structure. The scaling curves are presented over three different ranges in the phase-space distance $\tau$ since a single calculation could not span the various scaling regimes. For quite short time scales $\Delta t=10^{-9}$, the correlation dimension $\nu$ is effectively one, confirming the locally linear smooth phase-space path. For larger sampling rates $\Delta t=10^{-5}$, one sees a transition from $\nu=1$ to larger values, but there is no apparent scaling suggestive of the strange attractor. For still larger sampling rates, now comparable with the typical time scales of the attractor, one sees a convergence of the local slope for different embedding dimensions around the known value $D \approx 3.3$. This scaling regime is rather small, spanning just one order of magnitude in $\tau$.

Similar calculations at the noise strength $\epsilon=1.0$ are given in Fig. 2. There is a new scaling regime with $\nu \approx 2.0$ at the smallest time scales in panel (a). This indicates the finite fractal dimension of Brownian motion. This scaling regime changes smoothly onto the one of Fig. 1(a), of locally linear orbits with $\nu=1$. At still larger time scales, we see that the correlation dimension no longer converges for different embedding dimensions $e$, which is the hallmark of a higher dimensional process.

The most important point of these two figures is that the crossover from Brownian noise to deterministic behavior occurs at quite small time scales, and at quite small distances $\tau$ between points in phase space. This conclusion is qualitative in that the size of the scaling 
regimes will generally not be known a priori; the sizes will depend nontrivially on the noise strength $\epsilon$ and on the length of the time series $N$.

The difficulty of identifying all the relevant scaling regimes in dimension plots from time series sampled with a fixed time step motivated us to explore alternative ways to see this same structure. Towards this goal, we used an algorithm given by Higuchi [15,8] that estimates a length-based fractal dimension $D$ of the graph $x(t)$ of a curve. This dimension is more appropriate for stochastic, rather than deterministic, series with continuous curves giving a value $D=1$, and self-similar stochastic curves giving a value $D=1.5$, which is the value expected for Brownian motion [16]. For a chaotic time series and sampling rates much longer than the correlation time we expect and find that $D=2$ since the time series acts as a plane-filling curve. (We will discuss this point further in a future publication.)

For three different noise strengths $\epsilon$, Fig. 3 gives the local slope (Higuchi dimension $D$ ) of scaling relations as a function of time interval $\tau$; the symbol $\tau$ is not quite the same as that used in the Grassberger-Procaccia plots and here directly means a time interval, rather than a space-state interval. In Fig. 3(a), we see a result that is equivalent to that already observed in Fig. $\mathbb{1}(\mathrm{a})$, namely that there is a smooth functional dependence at small time scales, for which $D=1$, and this crosses over quite clearly to a chaotic regime of $D=2$ at time intervals of order the characteristic time of the attractor.

At moderate noise strengths such as $\epsilon=0.01$, there is a new scaling regime at the smallest time scales with $D=1.5$, arising from the Brownian motion of the Langevin path. This crosses over to $D=1$ at longer time scales and then to $D=2$ at still longer time scales, indicating the different kinds of dynamic structure present. For still larger noise strengths such as $\epsilon=1.0$, the scaling regime for Brownian noise increases at the expense of the linear regime. For extremely large noise strengths $\epsilon \gg 1$, the deterministic structure becomes a small perturbation of the Brownian noise and only the $D=1.5$ scaling regime should be observable.

From the results summarized in these figures, we suggest that continuous-time processes arising from nonlinear Langevin equations - a case that models dynamical experiments per- 
turbed by external noise - lead to easily separated fractal-dimension scaling regimes corresponding to stochastic Brownian motion at short time scales and deterministic chaos at longer time scales. This generalizes the earlier calculations of Mizrachi et al and of Provenzale and Osborne.

\section{ACKNOWLEDGMENTS}

This research was supported by NSF grant ASC-8820327, and by an allotment of CRAY YMP time through the North Carolina Supercomputing Center. We are grateful to James Theiler for providing a copy of his box-accelerated dimension code, and to NCSA for making public their HDF libraries and visualization tools. 


\section{REFERENCES}

* Also Department of Computer Science

[1] Peter Grassberger, Thomas Schreiber, and Carsten Schaffrath, Int. J. of Bifurcation and Chaos 1, 521 (1991).

[2] Filipe J. Romeiras, Celso Grebogi, Edward Ott, and W. P. Dayanwansa, Physica 58D, 165 (1992).

[3] A. Brandstäter, J. Swift, H. L. Swinney, A. Wolf, J. D. Farmer, E. Jen, and J. P. Crutchfield, Phys. Rev. Lett. 51, 1442 (1983).

[4] Pierre Bergé, Yves Pomeau, and Christian Vidal, Order Within Chaos (John Wiley \& Sons, New York, 1984), p. 153.

[5] A. Ben-Mizrachi, I. Procaccia, and P. Grassberger, Phys. Rev. A 29, 975 (1984).

[6] A. R. Osborne and A. Provenzale, Physica 35D, 357 (1989).

[7] James Theiler, Phys. Lett. A 155, 480 (1991).

[8] N. P. Greis and H. S. Greenside, Phys. Rev. A 44, 2324 (1991).

[9] P. Grassberger and I. Procaccia, Physica D9 189 (1983).

[10] James Theiler, Phys. Rev. A 342427 (1986).

[11] Josep LLosa and Jaume Masoliver, Phys. Rev. A 425011 (1990).

[12] Edward N. Lorenz, Physica 13D 90 (1984).

[13] H. S. Greenside and E. Helfand, Bell System Tech. J. 601927 (1981).

[14] James Theiler, Phys. Rev. A 36(9), 4456 (1987).

[15] T. Higuchi, Physica 31D 277 (1988); Physica 46D 254 (1990).

[16] Jens Feder, Fractals (Plenum Press, New York, 1988), Chapter 10, p. 186. 


\section{FIGURES}

FIG. 1. Plots of the Grassberger-Procaccia dimension $\nu$ for a fixed zero noise strength $\epsilon=0$, and for three sampling rates of time series: (a) $\triangle t=10^{-9}$; (b) $\triangle t=10^{-5}$; (c) $\triangle t=10^{-2}$. The parameter $\tau$ here indicates a distance in a $e$-dimensional embedding space. For each sampling rate, a series of length $N=10^{5}$ was studied. The curves denoted by squares, crosses, and triangles correspond to embedding dimensions of $e=4, e=7$, and $e=10$ respectively.

FIG. 2. The same as for Fig. 1 except the noise strength has a finite value $\epsilon=1$. There is a scaling regime in (a) around $D=2$ which is typical of Brownian noise, while the deterministic scaling regime in (c) at larger time scales is no longer well-defined.

FIG. 3. Plots of the Higuchi dimension $D$ for numerical time series of the Langevin equation, Eq. 1. for noise strengths $\epsilon$ of: (a) $\epsilon=0.0$; (b) $\epsilon=0.01$; and (c), $\epsilon=1.0$. The parameter $\tau$ indicates the time interval separating pairs of points in a time series [15]. The curve in each panel is a union of data obtained from three time series of $10^{6}$ points with sampling rates of $\triangle t=10^{-3}, 10^{-6}$, and $10^{-9}$, since a single time series was not adequate to span all the scaling regimes. 


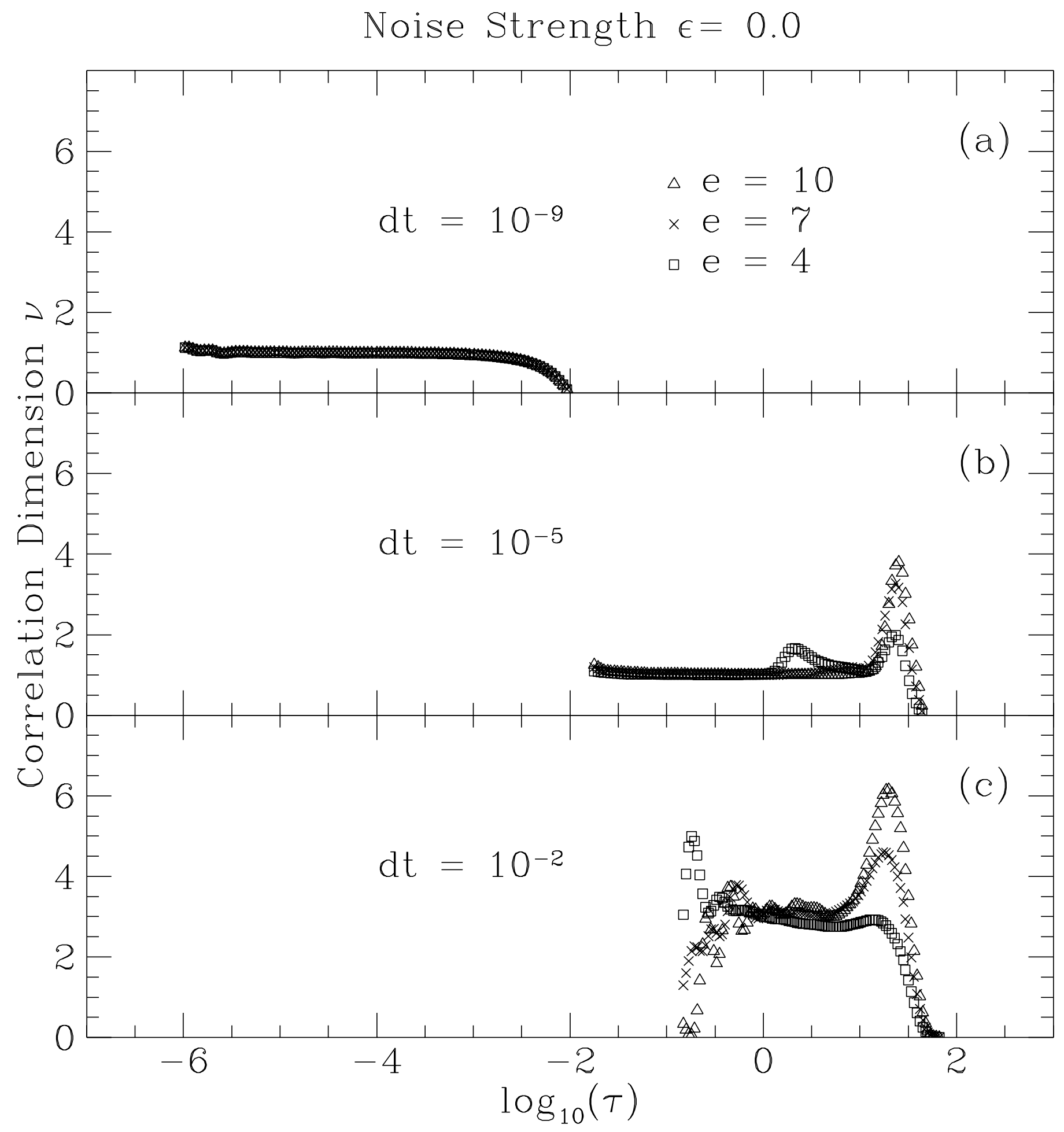

Figure 1 David A. Egolf and Henry S. Greenside, Phys.Rev.E, "Stochastic to Deterministic Crossover of Fractal Dimensions for a Langevin Equation" 


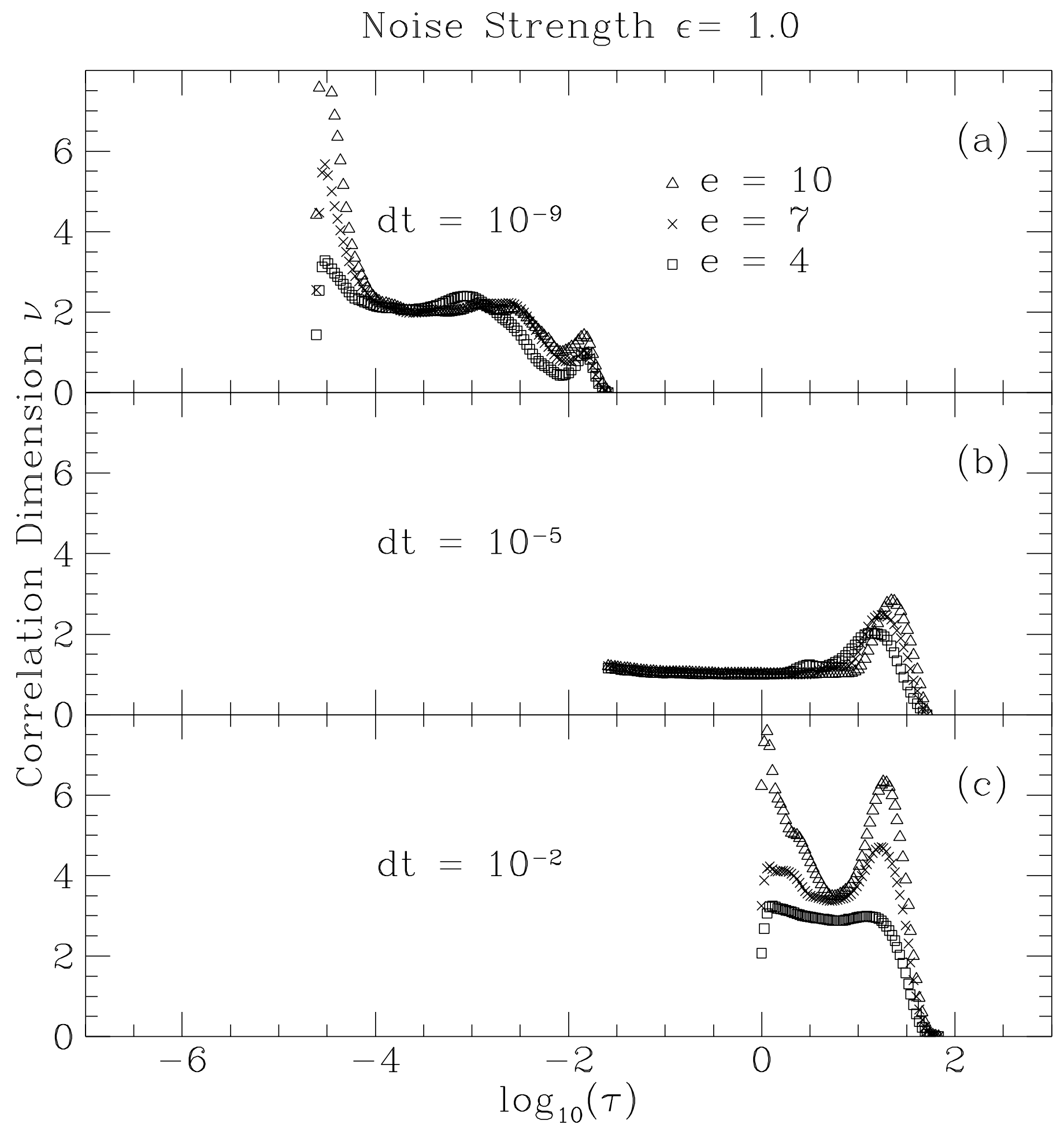

Figure 2 David A. Egolf and Henry S. Greenside, Phys.Rev.E, "Stochastic to Deterministic Crossover of Fractal Dimensions for a Langevin Equation" 


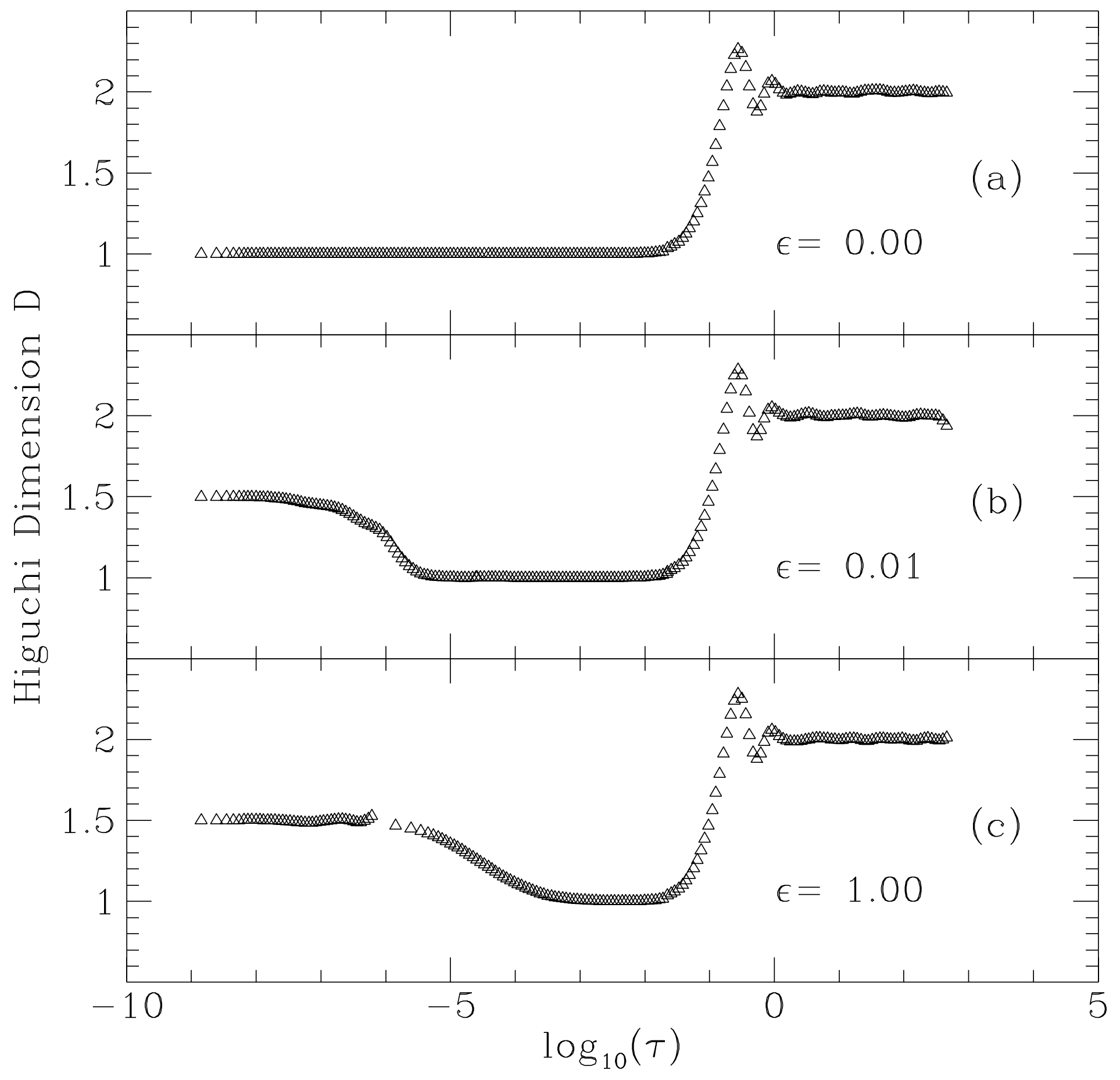

Figure 3 David A. Egolf and Henry S. Greenside, Phys.Rev.E, "Stochastic to Deterministic Crossover of Fractal Dimensions for a Langevin Equation" 